\title{
Formación en educación de los docentes clínicos de medicina
}

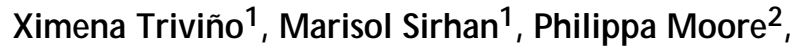 \\ Carlos Reyes ${ }^{3}$.
}

\section{Faculty development for clinical teachers in medicine}

Effective clinical teaching is an imperative of medical education. Clinical teachers and faculty development initiatives as well as Medical Schools, need to focus their efforts to pursue common outcomes: effective learning in students, future competent physicians and healthy patients. Excellence in quality of teaching needs scholars in education. To achieve this objective, institutions must recruit a core of medical faculty according to its mission, define the attributes and teaching competences, implement faculty development programs, and finally, support academic vitality. This article describes the three main issues of medical teaching: faculty, faculty development and its organization (Rev Méd Chile 2009; 137: 1516-22).

(Key words: Education, medical; Faculty, medical; Teaching)

Recibido el 16 de marzo, 2009. Aceptado el 13 de agosto, 2009.

${ }^{1}$ Centro de Educación Médica, ${ }^{2}$ Departamento de Medicina Familiar, ${ }^{3}$ Departamento de Medicina Interna, Escuela de Medicina, Pontificia Universidad Católica de Chile. Santiago de Chile.

“LA EDUCACIÓN SUPERIOR O UNIVERSITARIA ES DEMASIADO IMPORTANTE PARA DEJARLA/IMPARTIRLA AL AZAR ${ }^{\prime 1}$

L a excelencia en la calidad de la docencia clínica es fundamental para cumplir cabalmente con la misión de las Escuelas de Medicina. La formación de médicos competentes y responsables de brindar bienestar y cuidado integral a los pacientes depende en gran medida de alcanzar excelencia en la formación del cuerpo docente, que sea centrada en el estudiante y con una

Correspondencia a: Dra. Ximena Triviño Bonifay, M.H.P.E. Centro de Educación Médica, Escuela de Medicina, Pontificia Universidad Católica de Chile. Lira 44, Santiago Centro, Santiago, Chile. Tel.: (56-2) 3546972. Fax: (56-2) 6322802. E mail: xtrivino@med.puc.cl enseñanza de calidad que promueva el aprendizaje efectivo ${ }^{2}$. Una evaluación organizada y permanente de la calidad de la docencia clínica permite identificar fortalezas que deben mantenerse en el tiempo, detectar y remediar debilidades en aquellas áreas que deben ser corregidas y proponer recomendaciones de cambio para mantener altos estándares de calidad ${ }^{3}$.

La Comisión Ejecutiva de la Federación Mundial de Educación Médica (WFME) ha definido estándares internacionales para las Escuelas de Medicina en nueve áreas, destacando en relación a la docencia clínica que éstas deben asegurar que los estudiantes adquieran las competencias 
clínicas necesarias para desempeñar responsablemente la profesión, disponer de diferentes ámbitos clínicos para la práctica, y que los objetivos educacionales deben ser claramente compatibles con los principios de evaluación de los aprendizajes, métodos y prácticas, entre otros 4 .

Con respecto al cuerpo académico, recomiendan criterios de selección de los docentes, que incluyan la relación con la misión de la institución y méritos científicos y educacionales, el apoyo institucional para el desarrollo profesional y capacitación en docencia, y criterios de promoción definidos claramente. Por último, la monitorización y evaluación de los programas y cursos deben ser establecidas con mecanismos que aseguren una información permanente y confiable de la totalidad del sistema educacional para modificar los programas; dicha evaluación debe incluir la opinión de los estudiantes y profesores, el desempeño de los estudiantes, retroalimentación (feedback) de la evaluación de la información, información de los egresados, como también de la comunidad y la sociedad ${ }^{5}$.

Por otra parte, la Association of American Medical Colleges (AAMC) estableció cinco principios generales para lograr un nivel de erudición educacional (educational scholarship). Éstos incluyen: una definición apropiada de excelencia educacional, la práctica de actividades de apoyo al aprendizaje de excelencia, la evaluación de la docencia perfeccionada, el reconocimiento y recompensa de las actividades docentes, y por último, la existencia de una infraestructura organizacional de apoyo a los docentes ${ }^{6}$.

Estos principios, para lograr su desarrollo, se sustentan en tres pilares fundamentales y que corresponden a los ejes temáticos de esta revisión: el docente clínico, la formación en educación y una organización de apoyo a la docencia.

\section{EL DOCENTE CĹNICO}

La definición del perfil del docente clínico de excelencia y de las competencias que debe poseer es fundamental para guiar la formación en docencia. Se han planteado diversas proposiciones en la literatura internacional, algunas de las cuales se mencionarán a continuación.
Basado en las opiniones de los docentes, Markert identificó once atributos que caracterizan un buen docente: posee vocación docente y gran entusiasmo e interés por enseñar, enfoca la docencia en el aprendizaje de los estudiantes, enseña a un alto nivel cognitivo que permita organizar y recordar en forma útil, facilita la participación de los estudiantes, posee una buena base de conocimientos, prepara el material docente adecuadamente, está siempre disponible para los estudiantes, es innovador, promueve el aprendizaje activo y el autoaprendizaje, y crea una atmósfera donde los estudiantes trabajan por motivación intrínseca?.

Por otra parte, McLean, basado en la opinión de los estudiantes, establece que un buen educador es un buen comunicador, accesible, comprensivo, dispuesto a ayudar, amistoso, experto en el conocimiento que enseña, sensible a las necesidades y problemas de los estudiantes, entusiasta e interesado en lo que enseña, paciente y tolerante, motivador, buen guía, y no enjuiciador ${ }^{8}$.

De una manera más organizada, destacan tres proposiciones que han servido como marco teórico a modificaciones educacionales y organizacionales, y han sido la base de diversos programas de capacitación docente.

En la primera, Harden y Crosby en 2000, identifican 12 diferentes roles del docente clínico, cuyo marco conceptual serviría para la evaluación de necesidades de los docentes en la implementación de un currículo, la selección y promoción académica de docentes, la organización de un programa de capacitación, la asignación de responsabilidades docentes y la evaluación del docente $^{9}$. Se mencionan a continuación:

- Planificador: planificador de curricula y organizador de cursos.

- Evaluador: evaluador del aprendizaje de los estudiantes y de los curricula.

- Facilitador: facilitador del aprendizaje y mentor.

- Modelo: modelo en la docencia y en el trabajo médico.

- Proveedor de información: expositor en clases y docente clínico o práctico.

- Desarrollador de recursos: productor de guías de estudio y creador de material educativo.

En la segunda propuesta, Hesketh estableció un marco conceptual para el desarrollo de la 
excelencia de los educadores clínicos, que incorpora los roles del docente clínico y 12 competencias relevantes en tres niveles: desempeño docente, aproximación a la docencia y profesionalismo ${ }^{10}$.

El desempeño docente comprende siete competencias que deben poseer los docentes: enseñar en el ámbito clínico, enseñar grandes y pequeños grupos, planificar el aprendizaje, facilitar y manejar el aprendizaje, desarrollar y trabajar con recursos de aprendizaje, evaluar el aprendizaje de los estudiantes, evaluar cursos y desarrollar investigación en educación.

La aproximación a la docencia incluye: actitudes apropiadas, éticas y legales, comprensión de los principios de la educación y habilidades en la toma de decisiones y educación basada en la evidencia.

El profesionalismo, que otorga la categoría de profesor profesional en el rol docente, establece dos competencias: el rol del docente inserto en el sistema de salud y la comunidad y el desarrollo personal en relación a la docencia.

Por último, The Stanford Faculty Development Program define al docente efectivo en el ámbito clínico, como aquel que establece un clima positivo de aprendizaje, controla la sesión educativa, comunica los objetivos, promueve la comprensión y retención del conocimiento, evalúa el desempeño de los estudiantes, promueve el feedback y facilita el autoaprendizaje ${ }^{11}$.

La definición de un núcleo de competencias que debe poseer el docente clínico permite establecer un eje vertebral que facilita enfocar las estrategias y acciones para alcanzar la excelencia tanto en las áreas de formación como de evaluación ${ }^{12,13}$. Es fundamental implementar un plan de monitorización y evaluación continua con instrumentos e indicadores apropiados para medir la calidad de la docencia, las competencias y los roles del docente clínico. Los instrumentos comprenden: informe de actividades y portafolio docente, autoevaluación, encuestas a los estudiantes, y específicamente observación de las actividades docentes, revisión del material elaborado e implementación de innovaciones educacionales.

En la Universidad de Illinois en Chicago (UIC) se identificaron ocho dimensiones consideradas importantes para la implementación de una evaluación y que involucran diversas fuentes: infor- mación descriptiva, autoevaluación docente, evaluación de los estudiantes sobre la docencia, evaluación por pares, observación de la docencia en la sala de clases y en el ámbito clínico, información sobre los estudiantes, egresados y otros, evaluación del aprendizaje de los estudiantes, y contribuciones a los programas educacionales ${ }^{14}$. Cabe destacar algunos instrumentos más novedosos tales como el portafolio docente, medición de resultados de logro a largo plazo y la evaluación docente estructurada objetiva (OSTE, "objective structured teaching evaluation") ${ }^{15}$.

\section{FORMACIÓN EN DOCENCIA}

La profesionalización de la docencia considera la formación y capacitación de los docentes en materias educacionales y es esencial para lograr un mayor desarrollo en el ámbito disciplinar de la Educación Médica. Se reconoce como uno de los componentes centrales para promover la enseñanza y el aprendizaje efectivo en los estudiantes $^{9,17-19}$.

En 1975, Gaff propuso que los programas de formación docente en educación universitaria debieran apoyar a los docentes en mejorar sus destrezas de enseñanza, diseñar mejores curricula o programas educacionales y participar en el desarrollo adecuado de una cultura institucional. Un año más tarde se difundió la primera definición de formación en docencia clínica, como todas aquellas actividades desarrolladas por las instituciones para apoyar a sus docentes en la consecución de sus roles educacionales ${ }^{16}$. Finalmente, la definición de Sheets y Schwenk es la que actualmente se emplea con mayor frecuencia: "Cualquier actividad planeada para mejorar el conocimiento y las destrezas consideradas esenciales para el desempeño docente de los integrantes de un departamento o programa de residencia (ejemplo: habilidades clínicas, para enseñar, administrar, investigar)"20. Más aún, el concepto de logro de la efectividad docente se propone para todos los niveles del continuo educacional, pregrado, postgrado y educación profesional continua.

Desde los albores de la Educación Médica, en la década de 1970, diferentes tendencias fundamentadas en las teorías del aprendizaje aceptadas, 
influyeron en su desarrollo y aplicación. Posteriormente emergen las teorías cognitivas, centradas en la construcción activa del conocimiento y de los principios del aprendizaje de adultos. En 1990, surgen las teorías del aprendizaje social que promueven la educación centrada en el estudiante, el docente como modelo y la educación médica continua, y desde 2000 en adelante se han desarrollado fuerte y profundamente los conceptos y modelos derivados del profesionalismo, las competencias y la educación médica basada en evidencia, entre otras ${ }^{16}$.

Las ideas derivadas de estas teorías, en gran medida, han sido utilizadas al interior de las Escuelas de Medicina para diseñar e implementar una amplia variedad de programas de formación docente, obedeciendo idealmente como respuesta a la identificación de sus necesidades ${ }^{21}$. Están aquellos con énfasis en la capacitación sobre técnicas metodológicas para mejorar la efectividad de la docencia a todo nivel, hasta los que incorporan como propósito central el formar investigadores en educación médica y líderes que faciliten un perfeccionamiento continuo. Más aún, estos programas en educación médica han sido desarrollados con distintos niveles de profundidad, desde actividades de orientación para los nuevos docentes hasta programas de magíster, con actividades individuales como institucionales ${ }^{10,22}$.

La literatura internacional facilita el conocimiento de la experiencia de programas de formación en docencia clínica como modelos para el logro de diversos beneficios, personales como institucionales ${ }^{23-25}$. Incrementarían la motivación, satisfacción y socialización de los docentes dentro de la institución, mejorarían el aprendizaje de los estudiantes y la satisfacción de los pacientes, promovería una cultura académica docente, de investigación, innovación y evaluación para revisión de los programas de estudio ${ }^{26}$.

Para diseñar e implementar un programa de formación docente exitoso, Steinert recomienda comprender la cultura organizacional e institucional, determinar objetivos apropiados y establecer prioridades, evaluar las necesidades para asegurar la relevancia del programa, desarrollar diferentes programas para responder a las diversas necesidades, e incorporar principios del aprendizaje de adultos y diseño instruccional ${ }^{27}$.
Estos programas incluyen la enseñanza de grupos grandes y pequeños, en ámbitos clínicos: intrahospitalario, ambulatorio y comunitario; implementación de sistemas de evaluación del aprendizaje de los estudiantes; planificación y evaluación de programas y curricula; liderazgo educacional, desarrollo organizacional y para el cambio; MBE, destrezas comunicacionales e interprofesionalismo; y -con menor énfasis- preparación y entrenamiento individual (coaching), apoyo y guía por un mentor (mentoring), aprendizaje autodirigido, toma de decisiones e instrucción computacional $^{24}$.

El modelo de Kern para planificar la docencia es el más adecuado para el diseño e implementación de programas de formación docente ${ }^{28}$. Consta de seis etapas, cada una crucial para lograr el éxito. La primera es la identificación de la misión institucional y de las necesidades generales, la segunda es la evaluación de las necesidades de los docentes y el diagnóstico de la situación actual, la tercera es el establecimiento del propósito y los objetivos de aprendizaje, la cuarta es la selección de la metodología, la quinta es la implementación y la sexta es la evaluación del aprendizaje de los docentes-estudiantes, la evaluación del programa y seguimiento. No menos importante es que la planificación cuidadosa y la incorporación de estos críticos elementos en un programa educacional sirve de modelo para el diseño de los programas de curso en los cuales los docentes-estudiantes participan.

La última etapa, la evaluación del programa, es fundamental para obtener información que permita medir su impacto, tanto a nivel de los profesores como de los estudiantes, quienes van a ser los que demostrarán si el propósito de mejorar la educación y por ende el aprendizaje se ha logrado. En esta cadena de intervención, finalmente serán los pacientes quienes obtendrán mayores beneficios al ser atendidos en los campos clínicos de estas escuelas.

La evaluación de los programas de capacitación de los docentes es una tarea compleja, y los métodos utilizados para ello incluyen la evaluación del aprendizaje y encuestas de satisfacción al terminar cada curso y el programa completo a corto y largo plazo, pruebas 'pre' y 'post' concomitantes o retrospectivas para medir cambios cognitivos, de destrezas docentes y actitudinales 
(de actitud), autoevaluación de desempeño después de finalizada la capacitación, portafolio del programa, encuesta sobre estilos de enseñanza y observación directa de la conducta del docente entre otros ${ }^{29,30}$. La evaluación que los estudiantes y residentes realizan sobre la docencia y sus tutores aporta aún más información ${ }^{31}$.

El documento de la BEME (Best Evidence Medical Education) "Revisión sistemática de las iniciativas diseñadas para mejorar la enseñanza efectiva en la educación médica" recomienda el modelo de Kirkpatrick para la evaluación de los programas de formación y capacitación en educación médica ${ }^{32}$.

Consta de cuatro niveles de resultados: satisfacción de los estudiantes (respecto a la experiencia educacional), aprendizaje (cambios en actitudes, conocimientos y destrezas), conducta (cambios en la práctica y aplicación del aprendizaje a la práctica), y resultados (referencia a la mejoría del aprendizaje y el desempeño de los estudiantes o residentes como directo resultado de la intervención educacional y cambio organizacional). Sin embargo, dada su dificultad, la evaluación del impacto de estas actividades ha sido parcial hasta el momento.

El documento concluye que las actividades de desarrollo en docencia reportadas en la literatura aparecen altamente valoradas por los docentes. Que los participantes en general, refieren una alta satisfacción con estos programas, incorporación de nuevos conocimientos y destrezas educacionales, y cambios positivos en las actitudes (mayor confianza, motivación y entusiasmo), en su desempeño como educador, en la práctica organizacional y en el aprendizaje de los estudiantes. Sin embargo, existe escasa evidencia directa a nivel de resultados, tales como cambios en el sistema, de la práctica organizacional, 0 a nivel de los estudiantes, residentes o colegas.

En resumen, la evaluación permanente de los programas de capacitación en docencia y en consecuencia su perfeccionamiento y el alineamiento con las necesidades de las curricula de pre y postgrado aseguran en parte el éxito de la formación docente. La contraparte está dada por la motivación de los docentes, e íntimamente ligado a ello, el apoyo de las autoridades ${ }^{33}$.

\section{ORGANIZACIÓN PARA LA DOCENCIA}

El tercer pilar fundamental consiste en el establecimiento de una organización que apoya la docencia, denominación arbitraria para definir un "sistema operativo o estado de régimen" con una estructura organizacional articulada. Involucra el conjunto de acciones de soporte para la formación de los docentes y perfeccionamiento continuo de la docencia ${ }^{34,35}$.

Participan estamentos técnicos y directivos que fomentan una cultura institucional de excelencia académica.

Dado que el tratamiento de cada uno de estos componentes por separado reviste consideraciones particulares fuera del alcance y foco de esta revisión, a continuación se enumerarán de manera resumida los aspectos más importantes:

- La creación de unidades de Educación Médica (oficina, departamento o centros), así como instancias variadas de formación en educación deben dejar de ser consideradas una condición "de lujo" o de excepción ${ }^{16,26,32}$.

- La participación activa de los estamentos de liderazgo y autoridad institucional supone además del financiamiento, el sustento políticoacadémico de las intervenciones y estrategias en apoyo a la docencia al interior de la comunidad docente así como el establecimiento de políticas consensuadas ${ }^{36,37}$.

- Una cultura institucional que promueva, evalúe y recompense con incentivos la actividad académica educacional permanentemente. Considera la implementación de una carrera profesional académica en docencia y de un sistema que facilite el desarrollo académico mentoring y coaching. También un sistema de evaluación y calificación académica con normativas claras de promoción de la vía (track) preferente en docencia respecto de los tracks de investigación, actividad clínica, administración y extensión ${ }^{38}$. Por último, proporcionar incentivos para mantener la vitalidad académica con valoración del docente y su actividad.

- Red de comunicación efectiva y evaluación periódica de necesidades de los académi$\cos ^{39,40}$.

- Un enfoque de gestión centrado en el docente que incorpora motivadores extrínsecos (remuneraciones, beneficios de trabajo, reconoci- 
miento) e intrínsecos (logro de metas individuales, oportunidades de desarrollo, actividades sociales $)^{41,42}$.

- Uso eficiente de recursos limitados ${ }^{43}$.

\section{CONCLUSIÓN}

El sentido y el efecto de un programa educacional de formación de docentes clínicos sobrepasan los límites tanto individuales como institucionales. La sociedad y los individuos que la conforman finalmente son los mayores beneficiados, por lo que la recomendación para las Escuelas de Medicina es, sin lugar a dudas, evolucionar hacia la profesionalización de sus docentes ${ }^{44}$.

El suponer que el médico, por su calidad de tal, está capacitado para enseñar, y por ende, que sus estudiantes aprenden, es parte del pasado. El desafío es ir más allá; es imperativo contar con la implementación de programas adecuadamente es-

\section{REFERENCIAS}

1. BRown S. The institute for learning and teaching and UK approaches to accrediting teaching: looking to the future. Med Teach 2000; 22: 513-6.

2. Steinert $\mathrm{Y}, \mathrm{Mcieod}$ PJ. From novice to informed educator: the teaching scholars program for educators in the health sciences. Acad Med 2006; 81: 969-74.

3. Bland C, Wersal L, Vanloy W, Jacott W. Evaluating faculty performance: a systematically designed and assessed approach. Acad Med 2002; 77: 15-30.

4. WHO/WFME. WHO/WFME Guidelines for Accreditation of Basic Medical Education. Disponible en: http:// w w w.ifmsa.org/scome/wiki/ index.php?title=WHO/WFME Guidelines for Accreditation_of_Basic_Medical_Education [Consultado el 10 de enero de 2009].

5. WFME. WFME task force on defining international standards in basic medical education. Med Educ 2000; 34: 665-75.

6. Fincher R, Simpson D, Mennin S, Rosenfeld G, Rothman A, Cole M ET AL. Scholarship in teaching: an imperative for the $21^{\text {st }}$ century. Acad Med 2000; 75: 887-94.

7. MARKERT RJ. What makes a good teacher? Lessons from teaching medical students. Acad Med 2001; 76: 809-10. tructurados, basados en la evaluación de necesidades al interior de cada escuela y con estudio de su impacto.

Más aún, el desarrollo de diferentes modelos para optimizar el aprendizaje de los docentes, la formación de líderes educacionales, la promoción de cambios organizacionales y el establecimiento de redes de trabajo dentro y fuera de la propia institución, son las directrices futuras de las que estamos llamados a hacernos cargo ${ }^{45,46}$.

Agradecimientos

Agradecemos al Dr. Gonzalo Grebe, ex-Decano de la Facultad de Medicina de la PUC que formó una comisión de estudio de la Evaluación de la Docencia Clínica de Pregrado en la PUC en 2007 y a los integrantes del equipo académico: Drs. Eugenio Arteaga, Pablo Bertrand, Marcela Bitran, Ramón Corbalán, Patricio Giacaman, Sra. Francisca Márquez, Sra. Patricia Masalán y Dr. Arnoldo Riquelme.

8. MacLean M. Qualities attributed to an ideal educator by medical students: should faculty take cognizance? Med Teach 2001; 23: 367-70.

9. HaRDEN RM, CROSBY J. The good teacher is more than a lecturer: the twelve roles of the teacher. Med Teach 2000; 22: 334-47.

10. Hesketh EA, Bagnall G, Buckiey EG, Friedman M, Goodall E, HaRdEN RM et al. A framework for developing excellence as a clinical educator. Med Educ 2001; 35: 555-64.

11. SFDP. Stanford Faculty Development Programme. Disponible en http://www.stanford.edu/group/ SFDP/ [Consultado el 10 de enero de 2009].

12. Harris DL, Krause KC, Parish DC, Smith MU. Academic competencies for medical faculty. Fam Med 2007; 39: 343-50.

13. Purcell N, Loyd-Jones G. Standards for medical educators. Med Educ 2003; 37: 149-54.

14. The University of Illinois, College of Medicine. Teaching portfolio. Manual for evaluating teaching in the Collage of Medicine 2004. Disponible en http:// www.uic.edu/com/ $\mathrm{mcm} /$ teaching/COMTEACH.pdf [Consultado el 20 de enero de 2009].

15. Morrison H, Boker J, Howngshead J, Prisun M, Hitснсоск M, LUtzeiman D. Reliability and validity of an objective structured teaching examination for generalist resident teachers. Acad Med 2002; 77: S29-S32. 
16. McLean M, Cimers F, VAN Wyk J. AMEE Education Guide No 36: Faculty development: yesterday, today and tomorrow. Med Teach 2008; 30: 555-84.

17. IRBY DM. Clinical teacher effectiveness in Medicine. J Med Educ 1978; 53: 808-15.

18. IRBY DM. What clinical teachers in Medicine need to know? Acad Med 1994; 69: 333-42.

19. Rosselot E. Hacia el médico que nuestros países necesitan: énfasis en la comunicación y en la formación de los docentes. Rev Méd Chile 2003; 131: 331-7.

20. SHeETs KJ, Scwenk TL Faculty development for family medicine educators: an agenda for future activities. Teach Learn Med 1990; 2: 142-8.

21. McLeod PJ, Steinert Y, Meagher T, McLeod A. The $\mathrm{ABC}$ of pedagogy for clinical teachers. Med Educ 2003; 37: 638-44.

22. Robins L, Ambrozy D, Pinsky LE. Promoting academic excellence through leadership development at the University of Washington: the Teaching Scholars Program. Acad Med 2006; 81: 979-83.

23. Frohna AZ, Hamstra SJ, Mullan PB, Gruppen LD. Teaching medical education principles and methods to faculty using an active learning approach: the University of Michigan Medical Education Scholars Program. Acad Med 2006; 81: 975-8.

24. Gruppen LD, Simpson D, Searle NS, Robins L, Irby DM, MuLan PB. Educational fellowship programs: common themes and overarching issues. Acad Med 2006; 81: 990-4.

25. MuLER JH, IRBy DM. Developing educational leaders: the teaching scholars program at the University of California, San Francisco, School of Medicine. Acad Med 2006; 81: 959-64.

26. Davis MH, Karunathilake I, Harden RM. AMEE Education Guide $\mathrm{N}^{\circ}$ 28: The development and role of departments of medical education. Med Teach 2005; 27: 665-75.

27. Steinert Y, Cruess S, Cruess R, Snell L Faculty development for teaching and evaluating professionalism: from programme design to curriculum change. Med Educ 2005; 39: 127-36.

28. Kern DE, Thomas PA, Howard DM, Bass EB. Currículo development for medical education: a six-step approach. Baltimore: Johns Hopkins University Press; 1998.

29. BIERER SB, HuL AL. Examination of a clinical teaching effectiveness instrument used for summative faculty assessment. Eval Health Prof 2007; 30: 339-61.

30. McLeod PJ, Steinert Y, SNeLl L Use of retrospective pre/post assessments in faculty development. Med Educ 2008; 42: 543.

31. SteineRT Y. Using student focus groups to improve faculty performance. Acad Med 2001; 76: 574-5.

32. Steinert Y, Mann K, Centeno A, Dolmans D, Spencer J, Gelula M ET AL. A systematic review of faculty development initiatives designed to improve teaching effectiveness in medical education: BEME Guide No 8. Med Teach 2006; 28: 497-526.

33. Simpson D, Boyd R, Fincher RM, Rosenfeid G, Hafier J, Viggiano T ET AL. Advancing educators and education: defining the components and evidence of educational scholarship. 2007. Disponible en: http:// www .aamc.org/members/gea/ educ-scholar.pdf [Consultado el 20 de enero de 2009].

34. Thibault GE, Neill JM, Lowenstein DH. The academy at Harvard Medical School: nurturing teaching and stimulating innovation. Acad Med 2003; 78: 673-81.

35. Wiluams RG, Dunnington GL, Folse JR. The impact of a program for systematically recognizing and rewarding academic performance. Acad Med 2003; 78: 156-66.

36. Simpson D, Fincher RM, Hafler JP, Irby DM, Richards BF, RosenFeld GC eT AL. Advancing educators and education by defining the components and evidence associated with educational scholarship. Med Educ 2007; 41: 1002-9.

37. Oinonen MJ, Crowley WF, Moskowitz J, Vlasses PH. How do academic health centers value and encourage clinical research? Acad Med 2001; 76: 700-6.

38. Mark S, Lnk H, Morahan PS, Pololi L, Reznik V, TROPEZ, Sims S. Innovative mentoring programs to promote gender equity in academic medicine. Acad Med 2001; 76: 39-42.

39. Bland CJ, Seaquis E, Pacal JT, Center B, Finstead D. One school's strategy to assess and improve the vitality of its faculty. Acad Med 2002; 77: 368-76.

40. Demmy TL, Kiviahan C, Stone TT, Teague L, Sapienza P. Physicians' perceptions of institutional and leadership factors influencing their job satisfaction at one academic medical center. Acad Med 2002; 77: 1235-40.

41. Brawer J, Steinert Y, St-Cyr J, Waltersk, WoodDAUPHINEE S. The significance and impact of a faculty teaching award: disparate perceptions of department chairs and award recipients. Med Teach 2006; 28: 614-7.

42. Kumar A, Kalden DJ, Mathew T. Volunteer faculty: what rewards or incentives do they prefer? Teach Learn Med 2002; 14: 119-23.

43. CoHen JR, Fox S. Developing a new faculty practice plan with a model for funds flow between the hospital and the plan. Acad Med 2003; 78: 119-24.

44. Cruess SR, Johnston S, Cruess RL. Profession: a working definition for medical educators. Teach Learn Med 2004; 16: 74-6.

45. Steinert Y, Nasmith L, Mcleod PJ, Conochie L A teaching scholars program to develop leaders in medical education. Acad Med 2003; 78: 142-9.

46. Steinert Y, Cruess RL, Cruess SR, Boudreau JD, Fuks A. Faculty development as an instrument of change: a case study on teaching professionalism. Acad Med 2007; 82: 1057-64. 